\section{Patienten profitieren von konstanter Schmerzkontrolle}

Therapie der Wahl bei Tumorschmerzen sind nach der Praxisleitline der Deutschen Gesellschaft für Schmerztherapie (DGS) (http://dgs-praxisleitlinien.de) oral zu applizierende Substanzen der WHO-Stufen I-III, erklärte Johannes Horlemann, Geldern. Eine Präferenzsubstanz ist dabei Hydromorphon. Das Stufe-III-Opioid wirkt sowohl bei neuropathischen, nozizeptiven und viszeralen Schmerzen, so Horlemann. Vorteilhaft sei Hydromorphon insbesondere in der OROS ${ }^{\oplus}$-Galenik (Jurnista ${ }^{\varpi}$ ), die bei nur einmal täglicher Einnahme für eine kontrollierte, lang anhaltende Freisetzung sowie einen kontinuierlichen gleichmäßigen Plasmaspiegel über 24 Stunden sorge. Das vermeide End-of-dose-Schmerzen und das durchgehende Freisetzungsprofil erzeuge einen flachen Plasmaspiegel mit besserer Verträglichkeit.

Dagmar Jäger-Becker

Symposium: "Gemeinsam gegen den (Tumor-)Schmerz", Deutscher Schmerz- und Palliativkongress, Frankfurt/Main, 07.03.2013; Veranstalter: Janssen-Cilag

\section{Neuer Standard bei metastasiertem HER2+ Brustkrebs}

In ihren aktuellen Leitlinien empfiehlt die Kommission Mamma der Arbeitsgemeinschaft für Gynäkologische Onkologie (AGO) Pertuzumab (Perjeta ${ }^{\circledR}$ in Kombination mit dem bisherigen Standard Trastuzumab (Herceptin ${ }^{\circledR}$ ) plus Docetaxel als neuen Standard für die Erstlinientherapie des metastasierten HER2-positiven Mammakarzinoms. In dieser Indikation erhält der seit März 2013 zugelassene Antikörper den höchsten Empfehlungsgrad. Gleichzeitig spricht sich die AGO für die Anwendung des noch nicht zugelassenen Antikörper-Wirkstoff-Konjugats Trastuzumab Emtansin (T-DM1) im Rahmen klinischer Studien bei mit Trastuzumab vorbehandelten Patientinnen aus. red.

Nach Informationen von Roche

Follikuläre Lymphome

\title{
Subkutane Formulierung vereinfacht Therapie
}

\author{
Neuen Studiendaten zufolge ist der monoklonale Anti-CD20-Antikörper \\ Rituximab (MabThera ${ }^{\circledR}$ ) in subkutaner Formulierung vergleichbar sicher und \\ effektiv wie in intravenöser - bei deutlich kürzerer Behandlungsdauer.
}

Wirksamkeit und Sicherheit von Rituximab s.c. werden zurzeit in einer PhaseIII-Studie geprüft, an der 530 Patienten mit unbehandelten follikulären Lymphomen Grad 1-3a teilnehmen. Die Patienten erhalten randomisiert entweder 1.400 mg Rituximab s.c. oder $375 \mathrm{mg} / \mathrm{m}^{2}$ i.v., jeweils in Kombination mit einer CHOPoder CVP-Chemotherapie. Auch die Erhaltungstherapie wird mit Rituximab s.c. oder i.v. randomisiert fortgeführt. Die Daten der ersten 127 Patienten zeigten eine vergleichbare Bioverfügbarkeit und vergleichbare therapeutische Serumkonzentrationen von Rituximab s.c., so Ingo Schwaner, Berlin. 90,5\% der mit Rituximab s. c. behandelten Patienten erreichten Remissionen, davon 46,0\% eine kom- plette Remission. Bei i. v.-Gabe betrug die Remissionsrate 84,4\% mit 29,7\% Komplettremissionen. Beide Applikationsformen scheinen vergleichbar sicher, wobei es bei s. c.-Gabe nicht zu Infusionsreaktionen, sondern nur zu vorübergehenden lokalen Hautreaktionen an der Injektionsstelle kommt. Die Injektion s.c. verringere die Verabreichungsdauer von Rituximab auf 5-7 Minuten und verbessere so Patientenkomfort und Zeitaufwand für das Personal, betonte Study Nurse Julia Unkhoff, Münster. AbdolA. Ameri

Pressegespräch „ASH 2012: Dynamische Weiterentwicklung des Therapiestandards - Aktuelle Daten zum DLBCL und zu MabThera SC". Frankfurt/Main, 15.01.2013; Veranstalter: Roche

\section{Zielgerichtete Therapie bei NSCLC}

\section{Weitere Option erfolgversprechend}

Individualisierte Behandlungsoptionen gewinnen - gerade auch beim nichtkleinzelligen Lungenkarzinom (NSCLC) - weiter an Bedeutung. Afatinib ist ein neuer oral verfügbarer TKI, der die ErbB-Rezeptorfamilie hemmt.

Jürgen Wolf, Köln, stellte Daten der Studie LUX-Lung 3 vor, in der 345 Patienten mit fortgeschrittenem Adenokarzinom und EGFR-Mutation entweder Afatinib (40 $\mathrm{mg} / \mathrm{Tag}$ ) oder eine Chemotherapie mit Cisplatin und Pemetrexed erhalten hatten. Der primäre Endpunkt progressionsfreies Überleben war im Afatinib-Arm signifikant länger als im Chemotherapiearm (11,1 vs. 6,9 Monate; $\mathrm{p}=0,0004)$. Für Patienten mit den häufigen Mutationen del19 und L858R war der Vorteil mit 13,6 vs. 6,9 Monaten noch ausgeprägter [Yang JCH et al. ASCO 2012;Abstr. LBA7500]. Unter Afatinib zeigte sich zudem häufiger eine Verbesserung von Husten, Dyspnoe und Schmerz; auch die Lebensqualität stuften die Untersucher unter Afatinib höher ein [Sequist LV et al. ESMO 2012; Abstr.
1229PD]. Wolf betonte, dass alle Patienten mit einer Nicht-Plattenepithel-Histologie ihres Tumors auf EGFR-Mutationen getestet werden sollten, um gegebenenfalls eine Erstlinientherapie mit dem Tyrosinkinasehemmer zu ermöglichen.

Laut Pathologe Markus Tiemann, Hamburg, ist der Goldstandard bei der EGFR-Mutationstestung derzeit noch die Sequenzierung nach Sanger. In absehbarer Zeit würden jedoch Multiplex-Tests zur Verfügung stehen, mit denen eine gleichzeitige Testung auf unterschiedliche Mutationen möglich sei. Andreas Fischer

Meet the Expert "Therapieentscheidung beim fortgeschrittenen NSCLC - Welche Fragen stellt sich der Therapeut?" DGP-Kongress, Hannover, 21.03.2013; Veranstalter: Boehringer Ingelheim 